\title{
Special issue on the Biodiversity and Ecological Functions of Soil Fauna
}

\author{
Weixin Zhang, Shenglei Fu \\ Key Laboratory of Geospatial Technology for the Middle and Lower Yellow River Regions, Ministry of Education; College of Environment and \\ Planning, Henan University, Kaifeng 475004, China \\ (c) Higher Education Press 2021
}

Soil fauna are categorized into microfauna, mesofauna, and macrofauna, with tens of thousands of species for each kind. Soil fauna occupy different trophic levels in the soil food webs and play a critical role in soil processes, such as energy flow and matter transformation by mediating soil microbial community and trophic interactions. Soil fauna are sensitive to environmental changes and, therefore, can be used as bioindicators for environmental health. Besides, there is a great potential for the application of soil fauna in biodegradation and biomedication. Therefore, the diversity and ecological functions of soil fauna have attracted significant attention in recent years.

This special issue could not cover all the above-mentioned topics but aims at stimulating soil fauna-related research in China, which include several intersting aspects, such as the biogeographic distribution of arthropods and nematode, soil micro-food web response to grass mowing and nitrogen deposition, tillage-regulated earthworm contribution to root-derived carbon (C) incorporation into aggregates, and how interactions within soil micro-food webs or between different taxa of macrofauna could affect critical ecological processes.

As we stated ten years ago that, belowground ecology is booming in China, today it may keep pace with the developed world in some fields. Note that there were elaborated studies based on field soil fauna-manipulation experiment, mapping the regional-scale pattern of soil fauna distribution, applying isotope tracing technique to illustrate $\mathrm{C}$ transformation, and high-throughput sequencing to measure nematode diversity. Nevertheless, three cutting-edge aspects could be emphasized in future studies, i.e., 1) the precision manipulation of soil food webs, thus, understanding how soil food webs contribute to ecosystem services; 2) the changing pattern of soil faunaregulated ecological processes across spatial and temporal scales; 3 ) the coupling of soil fauna taxonomy databases derived from morphological analysis and DNA sequencing.

\section{Editors}

Prof. Shenglei Fu

Henan University, China

E-mail: fsı@henu.edu.cn

Prof. Weixin Zhang

Henan University, China

E-mail: weixinzhang@vip.henu.edu.cn 